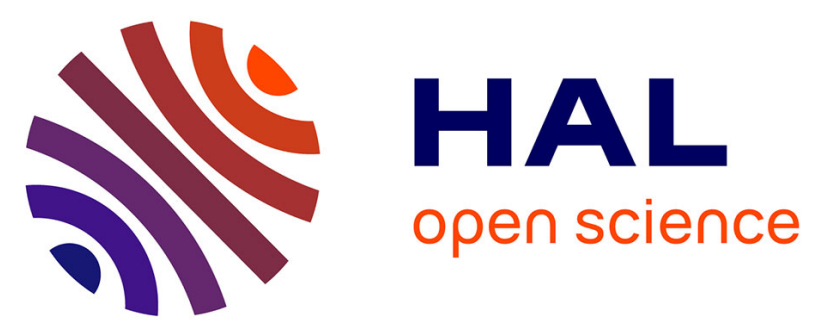

\title{
Coupling tomographic and thermographic measurements for in-situ thermo-mechanical tests
}

Léonard Turpin, Stéphane Roux, Olivier Caty, Sébastien Denneulin

\section{To cite this version:}

Léonard Turpin, Stéphane Roux, Olivier Caty, Sébastien Denneulin. Coupling tomographic and thermographic measurements for in-situ thermo-mechanical tests. Measurement Science and Technology, In press, 10.1088/1361-6501/abcc9f . hal-03025782

\section{HAL Id: hal-03025782 \\ https://hal.science/hal-03025782}

Submitted on 26 Nov 2020

HAL is a multi-disciplinary open access archive for the deposit and dissemination of scientific research documents, whether they are published or not. The documents may come from teaching and research institutions in France or abroad, or from public or private research centers.
L'archive ouverte pluridisciplinaire HAL, est destinée au dépôt et à la diffusion de documents scientifiques de niveau recherche, publiés ou non, émanant des établissements d'enseignement et de recherche français ou étrangers, des laboratoires publics ou privés. 


\title{
Coupling tomographic and thermographic measurements for in-situ thermo-mechanical tests
}

\author{
Léonard Turpin ${ }^{1,2,3}$, Stéphane Roux ${ }^{2}$, Olivier Caty ${ }^{1}$ \\ and Sébastien Denneulin ${ }^{3}$ \\ ${ }^{1}$ CNRS/CEA/SAFRAN/Univ. de Bordeaux \\ LCTS - Laboratoire des Composites Thermo-Structuraux, \\ F-33600, Pessac, FRANCE \\ ${ }^{2}$ Univ. Paris-Saclay/ENS Paris-Saclay/CNRS, \\ LMT - Laboratoire de Mécanique et Technologie, \\ F-91190, Gif-sur-Yvette, FRANCE \\ ${ }^{3}$ Safran Ceramics, F-33700, Mérignac, FRANCE \\ E-mail: leonard.turpin@ens-paris-saclay.fr
}

\begin{abstract}
In order to determine the thermo-mechanical properties of a complex $3 \mathrm{D}$ woven ceramic composite material, an experiment at high and inhomogeneous temperature and its dedicated full-field measurement procedure is developed. 3D tomographic images of the tested sample are captured at different stages of loading in a synchrotron beamline, and an infrared camera captures a side view of the sample as it rotates in the X-ray beam. A pin-hole projective model of the thermographic camera allows one to map the thermal field measured under numerous orientations onto a 3D mesh of the sample built from an initial tomographic image or a model. The projective model has to be calibrated, and an original procedure is proposed thanks to an integrated digital image correlation algorithm based on the "silhouette" of the sample (as only the protruding edges outlining the sample shape can be seen clearly). This procedure is illustrated with an experimental case study.
\end{abstract}

Keywords: Pin-hole projective model, Integrated-DIC, Infra-red imaging, In-situ thermo-mechanical test, Full-field measurement, Computed tomography 


\section{Introduction}

The use of in-situ mechanical tests in material science has substantially increased during past three decades. It consists in performing experimental tests (such as a mechanical test) inside a sophisticated piece of equipment delivering full field measurements, e.g. Transmission or Scanning Electron Microscopy (TEM or SEM) [1], Electron BackScatter Diffraction (EBSD) [2] or tomograph [3, 4]. Such experiments are quite complex to set up. Most of the time, a dedicated experimental device has to be developed. Providing exceptionally rich and highly resolved in space or time information about evolutions of the sample, they are among the most invaluable tools to understand and describe quantitatively material behaviour when subjected to a variety of different loadings including extreme conditions. The very last improvement consists in using multi-modality to further broaden in-situ measurement [5].

This work is focused on thermo-mechanical behaviour of thermo-structural composite materials [6]. Those materials are used at very high temperatures (more than $1000^{\circ} \mathrm{C}$ ), under severe thermal gradients, and yet, they are expected to preserve their structural qualities. Their very complex micro-structure produces anisotropic 3D thermal and mechanical fields calling for full-field measurements. Testing materials at such high temperatures is challenging and development of new experimental methodologies is needed $[7,8]$.

The originality of our experiments is that they are carried out using tomography and thermography. An in-situ loading device was designed to perform multi-modal experiments on complex-shaped samples [9]. The sample is heated externally in a range from $1000^{\circ} \mathrm{C}$ to $1200^{\circ} \mathrm{C}$ with a non-homogenous thermal field to induce elastic stress from kinematic incompatibilities so that damage induced by extreme thermal and/or mechanical loadings can be studied.

Tomography provides volume images of the sample. It is particularly relevant to study micro-structured materials or samples submitted to complex localized loading [10]. In-situ tomographic observation has first been developed to study damage and crack propagation [11]. It is the focus of most of those tests including the present study where the occurrence of damage is investigated.

Due to acquisition time, a set of tomograms is generally acquired at pre-defined loading steps. The acquisitions were done on steady states. After the thermal and mechanical loadings are applied, few minutes are waited for to let the temperature and damage fields reach a stationary state before the acquisition is ran. The comparison between two steps provides information on the evolution of the bulk of the sample. The bulk displacement field taking place between two acquisition steps can be computed from tomography using Digital Volume Correlation (DVC) $[12,13]$. In the global approach, DVC computation may be based on a Finite-Element (FE) mesh which can be built from a tomogram to reproduce accurately the sample geometry.

Infra-red (IR) thermography imaging is used in parallel to tomography. The thermal field emitted by sample surfaces is measured as the sample is rotated for the tomographic 
scan. Emissivity of the surfaces is uniform, and hence digital levels of the IR camera can be converted into temperatures. Moreover, because the tomographic scan is fast, the thermal inertia of the sample ensures that the temperature field is steady. Thus the temperature of the sample surface over most of the sample height can be estimated safely.

In order to exploit tomography and thermography jointly, it is necessary to associate the acquired raw data in a common framework. This requires a geometrical calibration of the IR camera that will account quantitatively for the sample geometry, described in 3D with a finite element (FE) mesh built from an initial tomogram, and captured at any rotation angle as a 2D image with the IR camera.

Such a geometrical calibration appears to share some similarities with that used in stereo-vision. The latter, a modern evolution of photogrammetry [14], consists in determining the 3D geometry of a scene from two (or more) 2D images, usually photographs, taken under several angles [15]. Calibration is a needed first step that aims to determine the optical parameters of the acquisition devices and their position on the scene. Calibration is typically performed thanks to a dedicated Digital Image Correlation (DIC) procedure using a textured (speckled) sample [16]. If the sample cannot be speckled, the calibration can be performed projecting a pattern on the object (e.g. fringe pattern [17]).

One specific difficulty of our experiment is that very few details of the sample surface can be tracked. Indeed, no speckle was used as a uniform emissivity allows an easy evaluation of temperature. Moreover, even edges between two visible surfaces cannot be clearly distinguished on the IR images. Hence only the sample protruding edges, namely the sample silhouette, can be trusted to calibrate the projective model. This very severe constraint leads to the development of an original methodology designed to be robust and accurate, to perform the geometrical calibration based solely on the sample silhouette.

Once the camera parameters are known, the projection operation is standard and can be used backward to transport the measured temperature field onto the mesh surface. Care has to be exercised to identify the visible surfaces - and to discard hidden ones - so that the thermal field can be mapped onto the visible parts of the sample surface.

An original procedure is proposed to couple those two full-field measurement modalities. After a brief presentation of the experimental setup, Section 2 details the formulation of the projective model problem and its calibration based only on the information available in thermographic images. In Section 3, this method is implemented and illustrated in an actual experimental case study. The filtering of the hidden sample surfaces is explained. The application is discussed in Section 4 together with the evaluation of the uncertainty of the thermal field. Finally, Section 5 sums up the main results and presents some future perspectives. 


\section{Methods: Calibration of projection matrix}

\subsection{Experimental setup}

A loading device was developed to perform tests on T-shaped or L-shaped samples of ceramic matrix 3D-woven composite materials [9]. It can be used both in laboratory or synchrotron tomography. The thermograms presented here were acquired during an experimental campaign at PSICHE beamline at synchrotron SOLEIL [18]. The mechanical quasi-static loading is applied step by step, alternating loading and tomographic acquisition (which takes about $15 \mathrm{~min}$ ). The geometry of the experimental configuration is schematized in Figure 1. The $\left(O, X_{t}\right)$ axis is the optical axis of the tomograph. The loading device and the sample are fixed on the tomograph rotating plate. They rotate with respect to the tomograph about $\left(O, Z_{t}\right)$ axis. During a tomographic acquisition, a large number of radiographs at different angles $\theta$ are taken. The angular step is sufficiently small so that the rotation of the plate can be continuous without degradation of the radiograph quality (no motion blur).

During the tomography, a series of thermograms is acquired with an IR camera which is positioned so that its optical axis $\left(T, Z_{c}\right)$ is oriented perpendicularly to $\left(O, X_{t}\right)$. This procedure provides a large set of view angles.

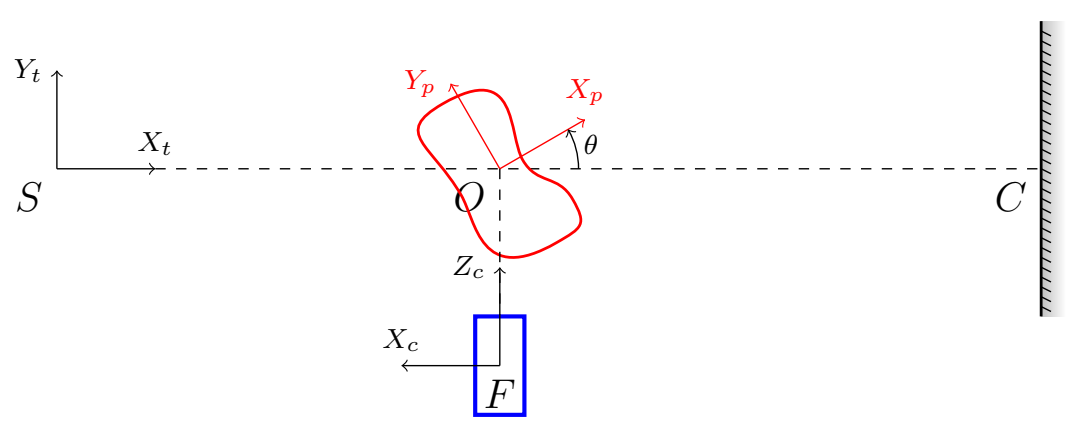

Figure 1: Scheme of the experimental configuration. The sample and the rotating stage are in red and the camera, in blue. $S$ is the X-ray source, $O$, the center of rotation and $C$, the center of the scintillator. $\left(O, X_{t}, Y_{t}, Z_{t}\right)$ is the coordinate system of the tomograph frame of reference. $\left(O, X_{p}, Y_{p}, Z_{t}\right)$ is the rotating plate frame. Finally $\left(F, X_{c}, Y_{c}, Z_{c}\right)$ denotes the frame of reference of the IR camera.

The objective is to use the information acquired by the IR camera as boundary conditions of a 3D FE model of the sample. The thermal field will be "reprojected" onto the 3D mesh surface using a pin-hole projective model. However, prior to this exploitation, it is necessary to calibrate the projective geometry, as detailed below.

\subsection{Stereo-correlation matrix decomposition}

The projection from the real space to the image space can be written as a projection matrix $[\boldsymbol{M}]$ which links the $3 \mathrm{D}$ homogeneous spatial coordinates of a point, $\{\boldsymbol{X}\}$, to 
the 2D homogeneous coordinates of its corresponding point, $\{\boldsymbol{x}\}$, in the image [19].

$$
\left\{\begin{array}{c}
s x \\
s y \\
s
\end{array}\right\}=[\boldsymbol{M}]\left\{\begin{array}{c}
X \\
Y \\
Z \\
1
\end{array}\right\}
$$

This formulation, where $s$ is a scale factor (which is the expression of the perspective effect), enables writing the projection as a linear problem. $[\boldsymbol{M}]$ is an a priori unknown $3 \times 4$ matrix. Due to $s,[\boldsymbol{M}]$ is defined up to a global magnification factor, so that eleven parameters remain to be determined.

This matrix has to be identified for the whole set of $n$ images. Without assumptions on the respective displacement of the object and the camera, $n$ projection matrices have to be computed. During the test, the camera if fixed and the sample, clamped to the tomograph rotating stage, undergoes only a rotation about the vertical axis. The stage movement is assumed to be accurate enough to rewrite the projection matrix $\left[\boldsymbol{M}^{i}\right]_{1 \leqslant i \leqslant n}$ for each angular position $\theta_{i}$, as

$$
\left[\boldsymbol{M}^{i}\right]=[\boldsymbol{M}]\left[\boldsymbol{R}^{i}\right]
$$

where $\left[\boldsymbol{R}^{i}\right]$ is the rotation matrix of angle $\theta_{i}$ around the tomograph axis. $[\boldsymbol{M}]$ has 11 parameters. The position and the orientation of the rotation axis of $\left[\boldsymbol{R}^{i}\right]$ is fully set with 5 parameters. The angular position of the first image can be arbitrarily set to $\theta_{1}=0$ (thus $\left[\boldsymbol{M}^{1}\right]=[\boldsymbol{M}]$ ), only $(n-1)$ unknown angular positions remain. For $n$ images, $n+15$ parameters are to be determined.

Any $3 \times 4$ matrix does not represent an admissible projection matrix. A way to take into account the constraints obeyed by $\left[\boldsymbol{M}^{i}\right]$ consists in decomposing it in elementary transformations (Figure 2) which can easily be written as linear transformations, and thus as matrices. Only $\mathcal{R}\left(\theta_{i}\right)$ depends on the considered image. $\mathcal{P}$ gathers intrinsic parameters which stand for camera optics. Extrinsic ones (i.e. related to the position of the object with respect to the camera) are decomposed to match the geometry of the considered experimental set-up.

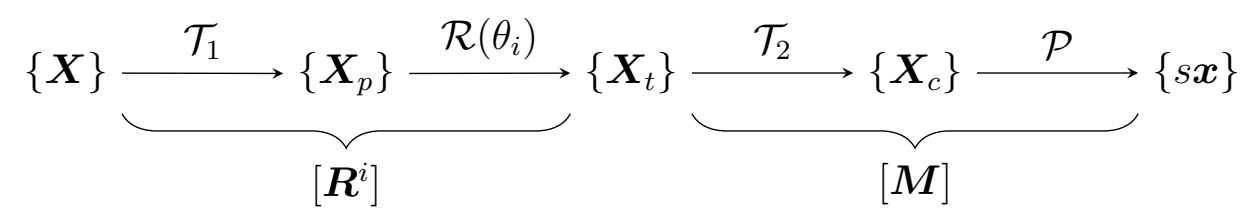

Figure 2: Decomposition of $\left[\boldsymbol{M}^{i}\right]$ into elementary transformations. $\mathcal{T}_{1}$ and $\mathcal{T}_{2}$ correspond respectively to the transformation from the $3 \mathrm{D}$ mesh frame to the tomograph rotating stage frame and from the tomograph frame to the camera frame. $\mathcal{R}\left(\theta_{i}\right)$ corresponds to the rotation of the stage. And $\mathcal{P}$ represents the projection onto the image plane.

This decomposition increases the number of parameters of the problem. Transformation $\mathcal{T}_{1}$ and $\mathcal{T}_{2}$ have 6 degrees of freedom (d.o.f.) (3 rotations and 3 
translations). For $\mathcal{T}_{1}$, as it just consists in aligning one of the basis axis with the tomograph rotation axis $\left(O, Z_{t}\right), 1$ translation is irrelevant. $\mathcal{R}\left(\theta_{i}\right)$ has only one d.o.f. $\theta_{i}$. And, without further assumption on the camera optics, $\mathcal{P}$ has 11 parameters. Finally $5+6+n+11=22+n$ parameters have to be evaluated.

As all the transformations $\mathcal{T}_{1}, \mathcal{R}\left(\theta_{i}\right), \mathcal{T}_{2}$ and $\mathcal{P}$ do not change during the experiment, calibration can be performed only once. Moreover, because a very stiff ceramic material was tested, the displacement of the sample under the mechanical load turned out to be very small (lower than thermogram resolution). Would the deformation of the sample be significant, it could be computed by DVC on tomographic images. The positions of 3D mesh (surface) nodes would then account for the deformed geometry. In fact, the only case which may be troublesome is when the sample slips out of its grip. If the sample requires a pre-loading for a good positioning, it is recommended to perform calibration only after the pre-loading step.

A gross projection matrix is first computed assessing the values of parameters. This matrix is used as an initialization for the digital image correlation procedure presented below.

\subsection{Shared information between $3 D$ mesh and thermograms}

Calibration consists in computing the projection matrix parameters in a given configuration (cameras and their respective positions and orientations). Usually this procedure operates on couples of images acquired by two different cameras [15, 20]. When images are not sufficiently textured for digital image correlation (DIC), a preliminary step using a dedicated calibration target is used. Such an approach determines the transformation between two images (1 and 2), $\left[\boldsymbol{M}_{2}\right]\left[\boldsymbol{M}_{1}\right]^{-1}$ which is not sensitive to some parameters which have to be set arbitrary as the 3D basis axes and a scale factor.

Here the exact transformations from the 3D frame used for the sample mesh and the image planes are sought. To make the correlation possible, data have to be pre-processed to be comparable (2D images). Moreover, thermography implies a homogeneous emissivity and grey levels are susceptible to vary in time so conventional calibration methods (based on sample texture) reveal improper [21]. The common information between the 3D mesh and the thermograms is thus the sample silhouette geometry. The projection of the protruding 3D mesh edges on 2D images (the silhouette) is compared to edges of the sample on the IR images. To do so, for each image, Integrated-DIC (IDIC) is performed between the silhouette of the mesh on the image plane and the binarized thermograms (Figure 3).

As the sample temperature is very high, the contrast between sample and surrounding is quite high, and binarization is easy. The grey level threshold is determined with an Otsu filter [22] but it could equally well be determined manually over a significant range without noticeable changes. This simple and classical procedure revealed satisfactory. In more complicated cases, images can be pre-processed to apply 
any needed correction (e.g. mask, more than one threshold, etc.).

The information supported by the edges of the silhouette of the sample is very poor for DIC, yet suitable for global DIC. Not using directly grey level field and hence being compatible with grey level changes expected for the thermographic images, it implies an enhanced robustness.

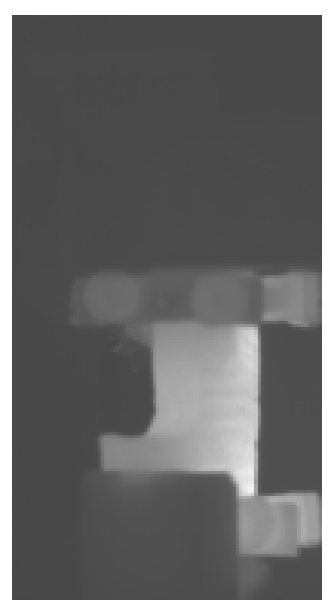

(a) Thermograph

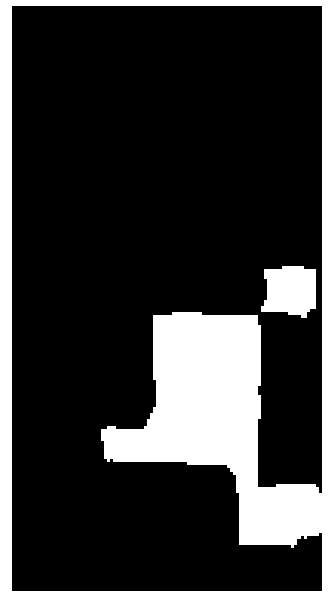

(c) Silhouette from the thermograph

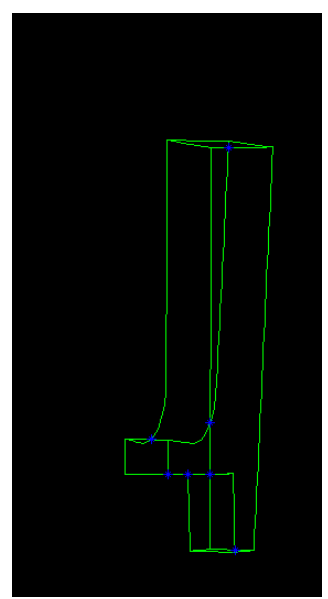

(b) Projection of 3D mesh outlines

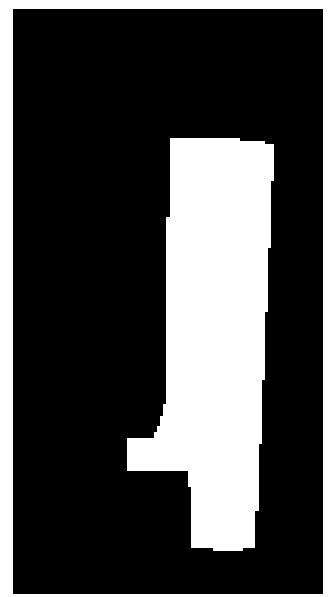

(d) Silhouette from the 3D mesh

Figure 3: Silhouettes of the sample are derived from either the thermograph (a) or a 3D mesh (b). From the thermograph, binarization is used to determine the silhouette from the thermograph (c), and edges extraction provides that of the mesh (d).

\subsection{IDIC procedure to determine matrix parameters}

DIC, is a full-field measurement procedure consisting in computing a displacement field $\boldsymbol{u}(\boldsymbol{x})$ between two images: the reference image, $f$, and a deformed one, $g$. Assuming grey level conservation, $\boldsymbol{u}(\boldsymbol{x})$ minimizes the residual field, $\rho(\boldsymbol{x})$,

$$
\rho(\boldsymbol{x}, \boldsymbol{u})=f(\boldsymbol{x})-g(\boldsymbol{x}+\boldsymbol{u}(\boldsymbol{x}))
$$


The global formulation consists in finding the field $\boldsymbol{u}$ minimizing the quadratic norm of the residual on the whole Region of Interest (ROI) [23].

$$
\Gamma(\boldsymbol{u})=\int_{\mathrm{ROI}} \rho(\boldsymbol{x}, \boldsymbol{u})^{2} \mathrm{~d} \boldsymbol{x}
$$

To do so, the displacement field $\boldsymbol{u}$ is progressively corrected by elementary corrections $\delta \boldsymbol{u}$ which minimize the linearization of $\Gamma(\boldsymbol{u})$ about the current point.

The main interest of such a formulation lies in the fact that $\boldsymbol{u}$ can be decomposed within any kinematic basis $\varphi(\boldsymbol{x})\left(\boldsymbol{u}(\boldsymbol{x})=\varphi_{i}(\boldsymbol{x}) a_{i}\right)$ - commonly, $\varphi(\boldsymbol{x})$ is a FE basis [24]. Finding $a$ is a non-linear problem which is classically solved from iterative corrections, computed for the tangent (and hence linear) problem. The incremental correction $\delta a$ minimizes $\widetilde{\Gamma}$

$$
\widetilde{\Gamma}\{\delta a\}=\frac{1}{2}\{\delta a\}^{\top}[H]\{\delta a\}-\{\delta a\}^{\top}\{b\}
$$

where $[H]$ is the Hessian matrix

$$
\left\{\begin{aligned}
{[H] } & =\int \varphi^{\top} \nabla f^{\top} \nabla f \varphi \mathrm{d} \boldsymbol{x} \\
\{b\} & =\int \varphi^{\top} \nabla f^{\top} \rho \mathrm{d} \boldsymbol{x}
\end{aligned}\right.
$$

The above minimization corresponds to solving the following linear system

$$
[H]\{\delta a\}=\{b\}
$$

and the correction $\{\delta a\}$ is to be combined with the current determination of the displacement $\{a\}$.

The displacement field $\boldsymbol{u}$ can depend on several parameters $\{p\}$. For each of them, $p_{i}$, the sensitivity field $S_{i}$ is defined as the ratio of the variation of displacement induced by an infinitesimal variation of $p_{i}$.

$$
S_{i}=\frac{\partial \boldsymbol{u}}{\partial p_{i}}
$$

Integrated-DIC consists in directly computing a vector containing the sought unknowns $\{p\}$. Instead of solving Eq. 7, the problem is reformulated as

$$
[S]^{\top}[H][S]\{\delta p\}=[S]^{\top}\{b\}
$$

where $[S]$ is the matrix concatenating all sensitivity fields of $\{a\}$ with respect to the parameters $\{p\}$ of $[\boldsymbol{M}]$. Passing through the intermediate basis $\{a\}$ is not mandatory but it simplifies the writing of the problem.

In the considered case, the unknowns are the geometrical parameters of $\left[\boldsymbol{M}^{i}\right]$ decomposition (Figure 2). The links between $\boldsymbol{u}$ and the parameters are explicitly known and the derivatives can easily be computed analytically. As an example, using the decomposition of Figure 2, $\left[S_{\mathcal{T}_{2}}\right]$ is

$$
\left[S_{\mathcal{T}_{2}}\left\{\boldsymbol{X}_{t}\right\}\right]=\left[\frac{\partial\left\{\boldsymbol{X}_{c}\right\}}{\partial\left\{p_{\mathcal{T}_{2}}\right\}}\right]=\left[\frac{\partial\left\{\mathcal{T}_{2} \boldsymbol{X}_{t}\right\}}{\partial\left\{p_{\mathcal{T}_{2}}\right\}}\right]
$$


The sensitivity of $\left[\boldsymbol{M}^{i}\right]$ w.r.t. the set of parameters of elementary matrix is written as a composition of elementary sensitivity matrix

$$
\left[S_{M^{i}}\{\boldsymbol{X}\}\right]=\left[\begin{array}{llll}
\mathcal{P} \mathcal{T}_{2} \mathcal{R} S_{\mathcal{T}_{1}}\{\boldsymbol{X}\} & \mathcal{P} \mathcal{T}_{2} S_{\mathcal{R}}\left\{\mathcal{T}_{1} \boldsymbol{X}\right\} & \mathcal{P} S_{\mathcal{T}_{2}}\left\{\mathcal{R} \mathcal{T}_{1} \boldsymbol{X}\right\} & S_{\mathcal{P}}\left\{\mathcal{T}_{2} \mathcal{R} \mathcal{T}_{1} \boldsymbol{X}\right\}
\end{array}\right]
$$

and the global sensitivity matrix, considering the full set of images is naturally

$$
\left[S_{M}\right]=\left[\begin{array}{c}
S_{M^{1}} \\
\vdots \\
S_{M^{N}}
\end{array}\right]
$$

2.4.1. Support of sensitivity fields As explained in section 2.3, the relevant information for DIC is located at the edges of the sample: in the area where the gradient of images (see Figure 3c-3d) is not null. So only the edges which lines up the silhouette (outline of the indicator function) are to be exploited. Moreover, to compute the sensitivity matrix, the coordinates of the corresponding points in the 3D domain have to be known. Thus only the projection of the protruding edges of the 3D mesh is to be considered.

2.4.2. Pyramidal approach to DIC The registration of the silhouette onto the thermographic image using DIC cannot be performed directly with binary images. Indeed, as seen in Eqs. 6-7, images are used only through their gradients, which are sparse and ill-behaved. The abrupt variations of grey levels at boundaries induce a "short-sightedness" of the algorithm. Intermediary steps to fix it consist in first blurring images using a convolution with a Gaussian of width $\sigma$ (Figure 4). In a pyramidal approach, several decreasing lengths, $\sigma$, allows one to solve Eq. 9 even if the initialization is quite far from the solution. Using first a large width $\sigma$ makes registration robust with respect to large displacements and then, at the end of the process, exactness is secured by not using any filter $(\sigma=0 \mathrm{px})$. It speeds up the resolution without prejudice to accuracy.
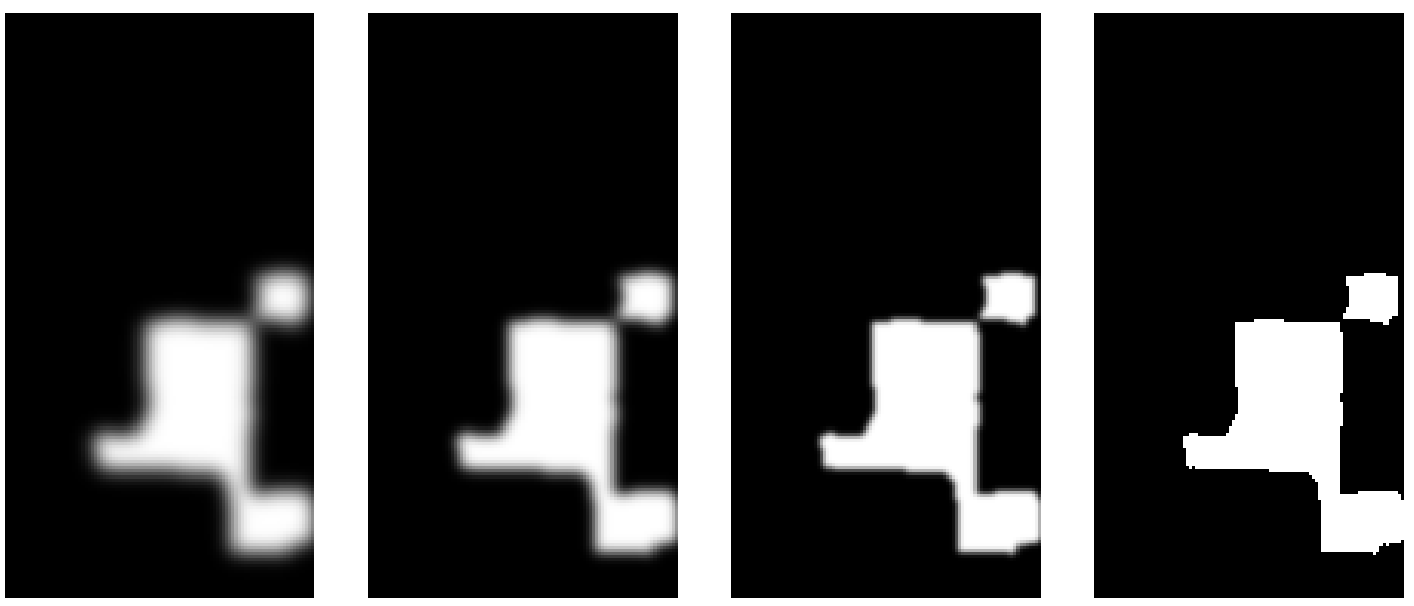

Figure 4: Blurred binarized thermograms used in the pyramidal approach, $\sigma=[3,2,1,0 \mathrm{px}]$ from left to right 
Two different convergence criteria are defined. The first one is based on the $\infty$ norm of incremental nodal displacement vector. When this criterion is met, the value of $\sigma$ is decreased to sharper gradients (and hence better accuracy) once the current registration is close to the sought solution. The second is the 2 -norm of the residual decrease rate and is only used at the end of the computation, at the lower floor of the pyramidal approach, where $\sigma=0$.

\subsection{Conditionning improvement}

With a single projection, it is obviously not possible to determine the parameters of the projective model. The problem is ill-posed. As for stereo-vision, one has to consider at least two different points of view. But even so, in our case because of the poor information content of the silhouette, the problem defined by Eq. 9 appears as illconditioned.

The definition of parameters $\{p\}$ involves the choice of parameters expressed in a specific frame. Although a mere translation and/or rotation does not change the nature of the problem, and hence not the conditioning of the Hessien, the inherent singularity of the Euler angles for an axis oriented close to the "north" pole is susceptible to make the problem artificially singular (the azimuth direction is undefined at the north pole). This is especially true in the present problem where the rotation axis of the tomograph appears to be a natural choice for the frame of reference. Hence it revealed convenient to rotate the frame within which the sample is described by a large angle to keep safe from this singularity.

Most of the parameters are rotations and translations. A second step is to normalize all rotation parameters (angles) with a characteristic length so that all variations of those parameters have about the same magnitude. Typically, this characteristics length is chosen equal to half the sample diameter.

Finally, when the above procedures are not sufficient, a Thikonov regularization is used [25]. It consists in adding the identity matrix scaled by a very small parameter to the Hessian. Here, this parameter is chosen to truncate the condition number (i.e. the ratio of maximal and minimal eigenvalues) at $10^{6}$.

\section{Application}

\subsection{Simplifying assumptions}

Additional assumptions on the problem geometry may help reducing the number of d.o.f. with no loss of accuracy. First, as the rotation speed of tomograph plate and the IR camera acquisition frequency are kept constant, the angular step between images is accurately known and so is $\theta_{i}$, for all $i$. The set of rotation transformations $\mathcal{R}\left(\theta_{i}\right)$ is fully determined. With this assumption, all parameters can be determined using two images. More images can be used to improve accuracy, computation time and the conditioning of the problem. 
$\mathcal{T}_{1}$ and $\mathcal{T}_{2}$ cannot be simplified further and require respectively 5 and 6 d.o.f.

At the resolution of thermograms, optical distortions are assumed to be negligible. Thus $\mathcal{P}$ depends only on 3 parameters: the focal length, $\lambda$, and a possible translation offset if the centre of the camera sensor is not centered on the optical axis.

$$
\mathcal{P}=\left[\begin{array}{cccc}
\lambda & 0 & 0 & t_{x} \\
0 & \lambda & 0 & t_{y} \\
0 & 0 & 1 & 0
\end{array}\right]
$$

Optical distortions are linked to intrinsic parameters of the camera. If they were not negligible, they could be determined once and for all before experiments with a dedicated calibration setup (as in [26] for example).

Eventually, 14 parameters are to be determined. In the hereafter discussed case study, eight images are used to perform the calibration.

\subsection{Mesh projection}

On the thermograms, only well-oriented surfaces (i.e. facing the camera) are visible. Building the projected mesh, the scalar product between the outgoing normal of each $3 \mathrm{D}$ surface element and the vector connecting the barycentre of the considered element to the focal point of the camera is computed (Figure 5b). Only elements for which this product is positive are considered (the other ones being obviously hidden).

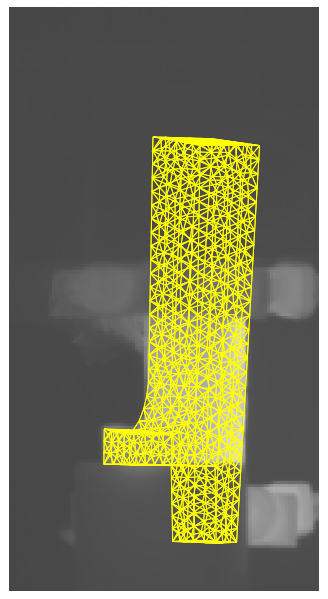

(a)

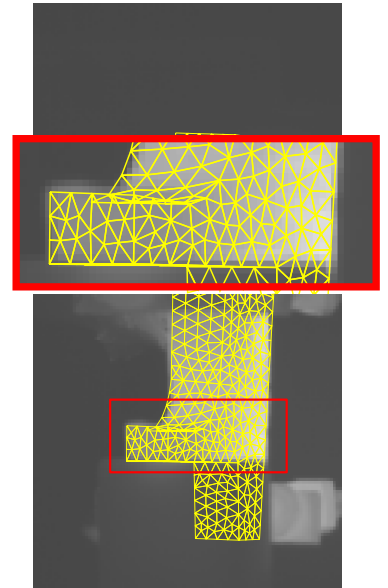

(b)

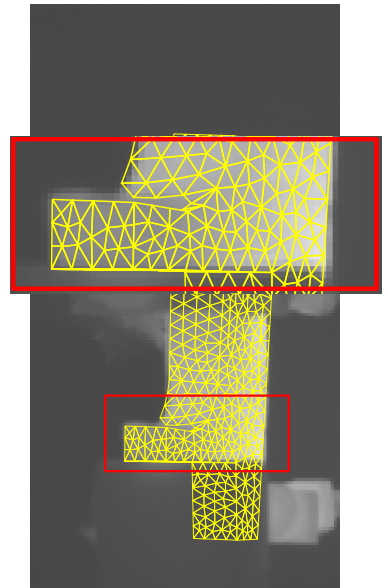

(c)

Figure 5: 3D Mesh projection (a) before filtering, (b) only well-oriented elements, (c) after filtering of hidden elements

Because the considered sample is not convex, this criterion is not sufficient to decide whether a surface element is visible or not because parts of the object can hide some well-oriented elements. All elements whose barycentre is hidden are considered to be hidden themselves. If the line segment connecting a barycentre $N$ of the $3 \mathrm{D}$ mesh element to its projection $P$ on the image plane crosses a $3 \mathrm{D}$ surface element then it is 
hidden. Efficient algorithms to compute the intersection of a segment line and a triangle have been developed for ray tracing. The approach followed here is a simplified version of the algorithm proposed by Moller et al [27].

Let $A B C$ be a $3 \mathrm{D}$ surface element and $I$ the intersection point of a ray $(P N)$ with the plane defined by $A B C$ (Figure 6 ), the vector $\overrightarrow{P I}$ can be decomposed as

$$
\overrightarrow{P I}=\lambda \overrightarrow{P N}=\overrightarrow{P A}+\xi \overrightarrow{A B}+\chi \overrightarrow{A C}
$$

Using Cramer's rule

$$
\left\{\begin{aligned}
\lambda & =\frac{\overrightarrow{P A} \cdot \vec{n}}{\overrightarrow{P N} \cdot \vec{n}} \\
\xi & =\frac{(\overrightarrow{P A} \wedge \overrightarrow{A C}) \cdot \overrightarrow{P N}}{\overrightarrow{P N} \cdot \vec{n}} \\
\chi & =\frac{(\overrightarrow{P A} \wedge \overrightarrow{A B}) \cdot \overrightarrow{P N}}{\overrightarrow{P N} \cdot \vec{n}}
\end{aligned}\right.
$$

where $\vec{n}=\overrightarrow{A B} \wedge \overrightarrow{A C}$.

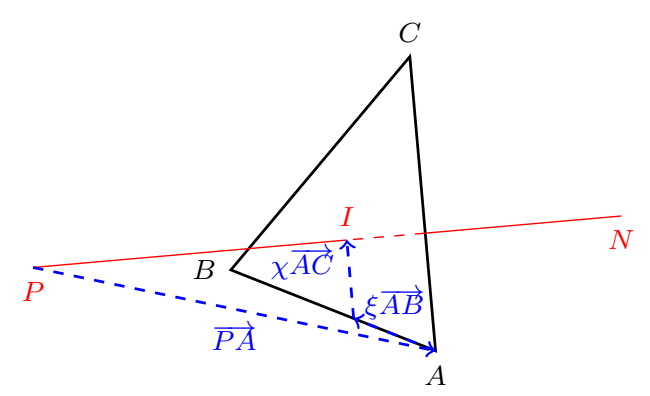

Figure 6: Decomposition of $P I$ in the basis defined by the triangular element $A B C$

The node $N$ is hidden by the element $A B C$ iff $I$ lies in $A B C$ and $I \in[P N]$. This provides five conditions on the parameters

$$
\left\{\begin{array}{c}
0 \leq \xi \text { and } 0 \leq \chi \\
\xi+\chi \leq 1 \\
0<\lambda<1
\end{array}\right.
$$

Not to be sensitive to computational errors, a tolerance $\varepsilon$ is integrated such that $0<\varepsilon \ll 1$

$$
\left\{\begin{array}{c}
\varepsilon<\xi \text { and } \varepsilon<\chi \\
\xi+\chi<1-\varepsilon \\
\varepsilon<\lambda<1-\varepsilon
\end{array}\right.
$$

This system has to be solved for each couple node-element, its crude implementation needs two nested loops. To easily bypass this problem and to drastically reduce computation time, the projection on the focal plane of the camera is considered. The 
projection of every node coincides with the focal point: $\forall N, P=F$. Decoupling the vectors linked to the nodes and the ones linked to the element, the computation of coefficients $\lambda, \xi$ and $\chi$ is reduced to matrix products. The three matrices (nb. of nodes $\times$ nb. of el.), corresponding to the three coefficients enable to find all the nodes which respect the conditions given in Eq. 17. Figure 5c shows remaining elements.

\subsection{Thermal field mapping}

Using a prior black body calibration, the thermogram grey levels are converted to an equivalent temperature. This temperature field can then be re-projected onto the visible surfaces of the 3D mesh, and affected to the mesh nodes. Assuming the temperature field to be stationary in time, each nodal temperature value is averaged in time (over the frames where the node is visible).

Assuming further the temperature field to be spatially smooth enough to be faithfully described by a 3D polynomial field, the previous determination is projected onto such a polynomial field thanks to a least squares regression,

$$
T(\boldsymbol{X})=\operatorname{argmin} \sum\left(T(\boldsymbol{X})-\frac{\sum_{\theta} \delta(\theta, \boldsymbol{X}) f(\boldsymbol{x}(\theta))}{\sum_{\theta} \delta(\theta, \boldsymbol{X})}\right)^{2}
$$

where $\{\boldsymbol{x}(\theta)\}=[\boldsymbol{M}(\theta)]\{\boldsymbol{X}\}, \delta(\theta, \boldsymbol{X})$ is equal to 1 or 0 , respectively when the node $\boldsymbol{X}$ is visible in the image under the angle $\theta$ or not. This additional step further filters out small fluctuations that resulted from the previous mapping. The difference between the two temperature fields allows one to assess the unphysical nature of the filtered fluctuations, and its very small magnitude.

\section{Result and discussion}

\section{1. "Re-projecting" thermal field}

Figures $7 \mathrm{a}$ and $7 \mathrm{~b}$ present the projection of the $3 \mathrm{D}$ mesh in a thermogram with, respectively, the initial estimation of parameters and their fine calibration. In this example, the initialization of parameters is estimated from the nominal position of the sample with respect to tomograph rotating stage (i.e. the sample is vertical and centered on the rotation axis), and the position of the camera (as in Figure 1). The scale of the sample is rather well-estimated. The calibration algorithm efficiently corrects angular and positioning errors. After calibration, projected (2D) meshes fit accurately the sample in the IR images.

Camera calibration problem is ill-conditioned and the convergence is typically very slow (a fortiori when the calibration is based only on few reference points, as in manual procedure). So the number of iterations at convergence depends much on the value of convergence criterion. Here the conditioning improvement (part 2.5) and the relevant amount of data provided using the full edges of the sample and 8 images for calibration drastically reduce the number of iterations (22 iterations for the presented case). Each 
iteration is more complex and more time-consuming but the overall procedure remains fast without any computational optimisation.

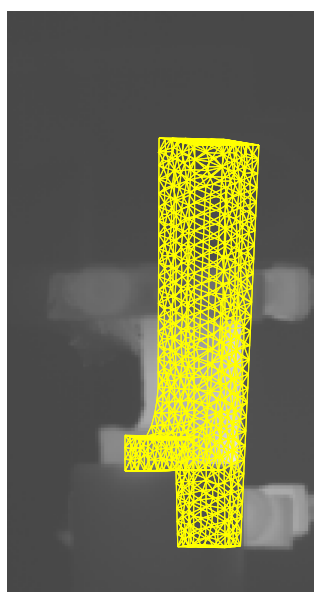

(a)

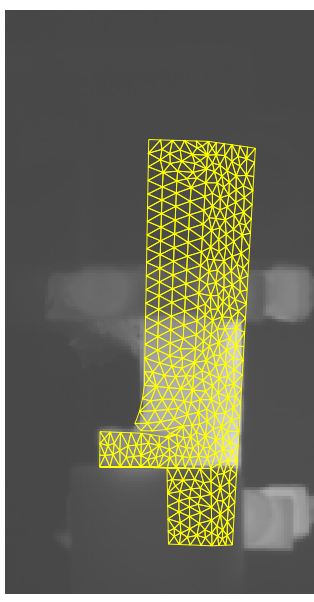

(b)

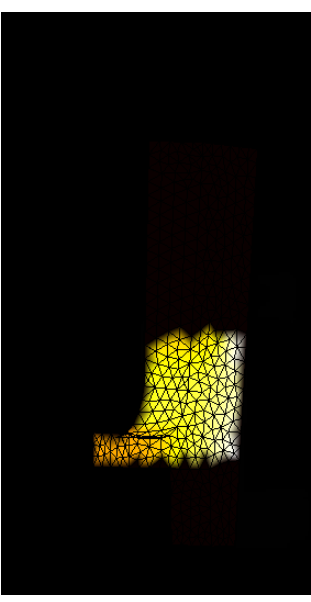

(c)

Figure 7: Result of calibration and affectation of temperature values onto the surface mesh (a) before calibration, (b) after calibration, (c) temperature mapping

The temperature field is expressed at the nodes of the 2D mesh (Figure 7c). Due to interpolation, the temperature close to the protruding edges is distorted. During the computation of 3D field, the nodes of those edges are omitted. However, the information provided by the whole set of images is redundant enough to allow for the determination of a 3D polynomial fit (here a degree 2 revealed sufficient) of the temperature data to be performed accurately, and hence the full 3D surface temperature map can be obtained (Figure 8a).

After calibration, the temperature was followed in time over the observed surfaces. Even if the temperature field is not strictly stationary - as the sample is rotated on the tomographic turn-table - the temperature change revealed hardly measurable because of the thermal inertia of the sample. Regarding the uncertainty on the measured field, the thermal field can be considered stationary.

This 3D field can effortlessly be mapped from a mesh to another. As an illustration, the mesh of Figure $8 \mathrm{~b}$ corresponds to the part of the sample where the interpolation is relevant (that is the part which is visible on IR images). Setting the thermal field re-projection procedure within the wider framework of inverse measurement, the field should be mapped on the mesh used for DVC.

\subsection{Considerations about errors and uncertainties}

4.2.1. Diffuse emissivity assumption The thermal field is diffuse, indeed the edges between two visible faces of the sample cannot be distinguished in any IR image. Moreover the grey level value of a physical point of the sample does not vary from one frame to the next. The emissivity of the sample seems not to depend on the view 


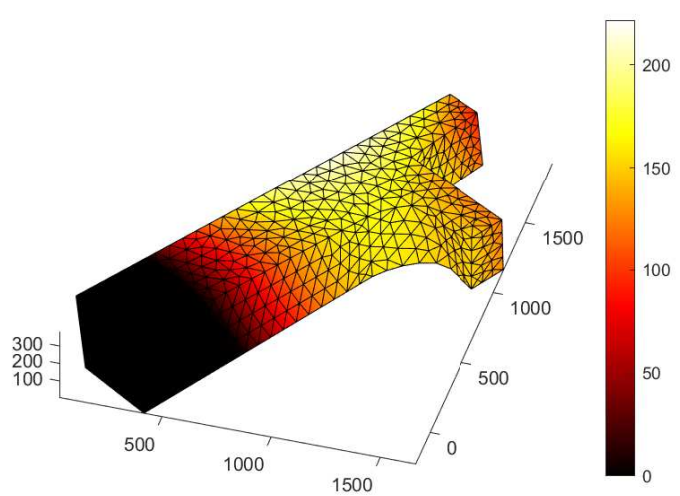

(a)

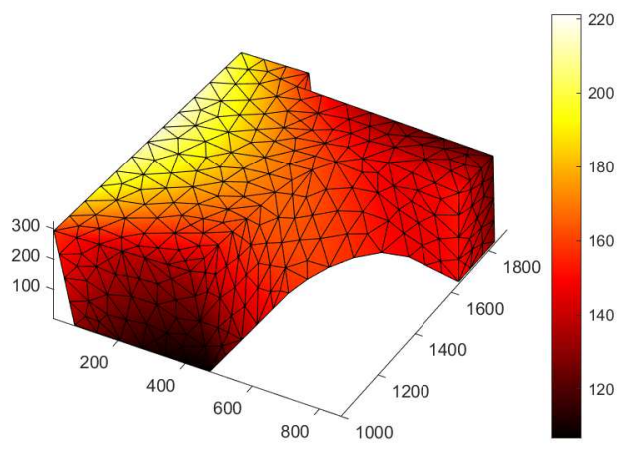

(b)

Figure 8: Registration of thermal field in 3D mesh (digital level) (a) on calibration mesh, (b) on another (finer) mesh

angle, thus, it is legitimate to use the camera software to convert the luminance data of the camera to temperatures.

4.2.2. Uncertainty induced by thermograms noise The uncertainty on the re-projected thermal field induced by IR images noise can be estimated empirically. IR-image noise level $\eta$ is computed as one half of the standard deviation of the difference between two images of the same scene. $\left(I_{i}\right)_{i=1 \ldots n}$ denotes the original set of IR images. A new set of images $\left(J_{i}\right)_{i=1 \ldots n}$ is built adding a Gaussian white noise of intensity $\eta$ to each images $I_{i}$. The two $3 \mathrm{D}$ thermal fields, $T_{I}$ and $T_{J}$, corresponding respectively to set $\left(I_{i}\right)$ and $\left(J_{i}\right)$ are computed. The error field $E$ is the difference

$$
E=T_{J}-T_{I}
$$

The uncertainty is characterized by the standard deviation of $E$. It is dependent on the artificial noise realizations. A large number of samples has to be used to estimate the average standard deviation $e$.

In our images, the noise level is $\eta=0.25 \%$ (of the full range of grey level). The 3D fields are computed onto the visible (meshed) surface of the sample (Figure 8b). Figure 9a shows an example of error field whose intensity remains low. It is quite smooth because it is the difference between two polynomial fields. The uncertainty $e$ is computed over 100 realizations. The graph (Figure 9b) presents the influence of the number of images used for the computation on the average uncertainty $e$ (the minimum and maximum uncertainties on the samplings are plotted in dotted lines). As expected, $e$ decreases with the number of images used. When the full set (48 images) is used, the uncertainty, induced by the noise affecting the IR images, is about $0.01 \%$.

4.2.3. Residual fields To evaluate the relevance of the $3 \mathrm{D}$ polynomial interpolation, residual fields are computed. They are a set of $2 \mathrm{D}$ images defined as the difference 


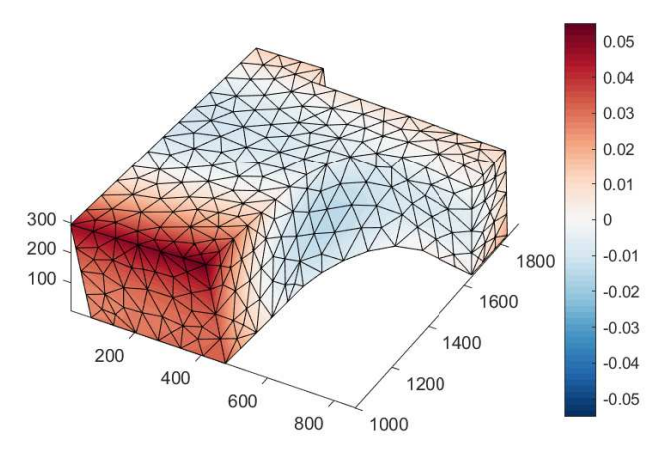

(a) $E(\%)$

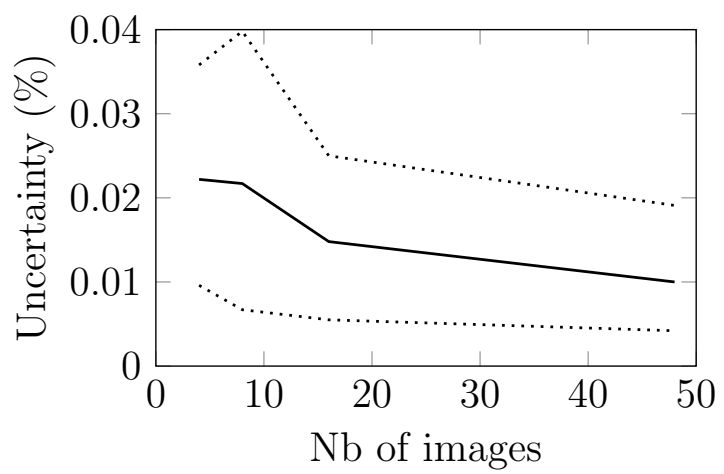

(b)

Figure 9: Estimate of the uncertainty of the re-projected field: (a) an error field; (b) average uncertainty $e$ is shown as a solid curve, whereas minimum and maximum uncertainties on the 100 independent samplings are indicated as dotted curves.

between the projection of the 3D polynomial field and the IR images (Figure 10). Their intensities are low (a few percent of image dynamic). As expected, the polynomial approximation smoothens the field and some differences are visible, namely near the edges of the sample. The luminance data on the thermograms can be affected by the environment of the sample. Taking into account the whole set of images, this effect is averaged out in the 3D field.

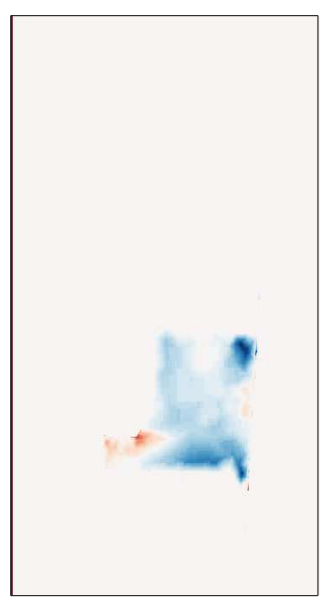

(a)

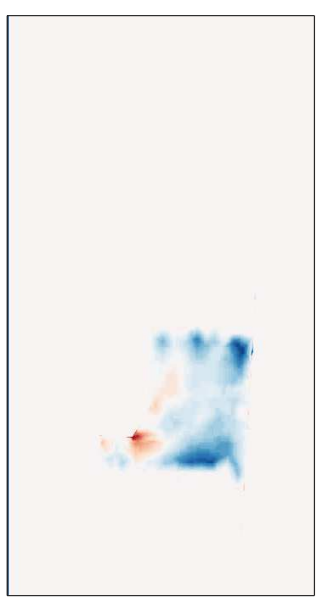

(b)

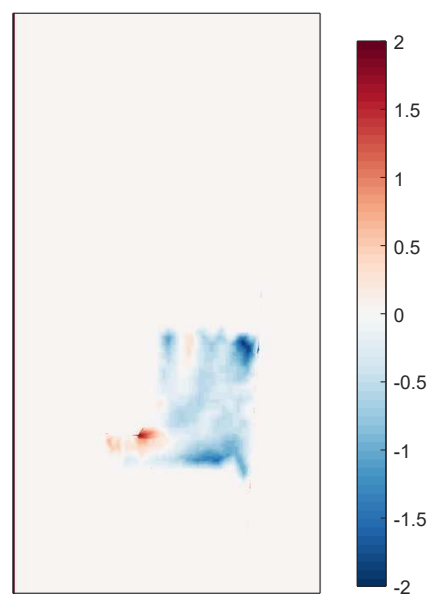

(c)

Figure 10: Residual (\%): Difference between the projection of the 3D polynomial field and infra-red images evaluated for polynomials of degrees 2, 3 and 4, respectively for (a), (b) and (c)

Residuals were computed for several polynomial fields of different order. All three have the same order of magnitude. For degree 2, 3 and 4 their norms are respectively $1.20 \%, 1.22 \%$ and $1.12 \%$. The assumption on the smoothness of the temperature field to be described by a low order polynomial approximation is deemed validated. 


\section{Conclusion}

To draw an intimate connection between two full-field measurement modalities, a new calibration procedure is introduced. In order to allow a direct use of the IR image intensity to infer the temperature value, the sample is not speckled. Hence, the calibration has to rely only on the silhouette of the sample. The proposed procedure is based on the correspondence of the protruding edges of the 3D mesh projected on the IR-camera image plane, and the outline of the sample extracted from the thermograms. The use of the calibrated projective model allows the temperature field to be mapped onto the surface of the 3D mesh of the sample, and to be readily accessible for any further thermo-mechanical modelling.

Up to now, in-situ thermo-mechanical tests within a tomograph, were carried out either at room or at high temperatures, but most of the times with a spatially uniform temperature field. However, for ceramic materials and especially ceramic composites it is very important to assess the material resistance to extreme temperature gradients potentially combined to mechanical loads. Moreover samples with a complex shape (such as T- or L-shaped woven composites) is required to obtain meaningful results, representative of extreme conditions that industrial parts have to sustain in service.

The proposed procedure allows to quantify thermal fields on the actual sample 3D geometry. Coupled with tomography and DVC, both temperature and displacement field can now be accessed with a consistent description, that of a 3D mesh of the sample, directly amenable to quantitative comparison with a numerical modelling. This opens up the possibility to validate complex thermo-mechanical model of complex microstructures with local highly anisotropic properties.

The presented procedure is quite generic and can be extended to other acquisition modalities. It can provide different fields, mapped onto the same frame, such as the temperature field in the present case, but it is also applicable to multiscale or multiresolution acquisitions.

\section{Acknowledgments}

L. Turpin $\mathrm{PhD}$ is supported by a convention CIFRE implying Association Nationale de la Recherche et de la Technologie and Safran Ceramics. Safran group and Direction Générale de l'Aviation Civile are acknowledged for funding this work through PRC MECACOMP. In-situ experiments were carried out at PSICHE beamline at synchrotron SOLEIL (Saclay, France) with the valuable support of Andrew King and were part of proposal 20181639. It is a pleasure to acknowledge David Damiani for the numerous discussions about infra-red thermography and his help during experiments. 


\section{References}

[1] M. Legros, D.S. Gianola, and C. Motz. Quantitative In Situ Mechanical Testing in Electron Microscopes . MRS Bulletin, 35(5):354-360, 2010.

[2] Stuart I. Wright, Matthew M. Nowell, and David P. Field. A review of strain analysis using electron backscatter diffraction. Microscopy and Microanalysis, 17(3):316-329, 2011.

[3] David R. Merrifield, Vasuki Ramachandran, Kevin J. Roberts, Wesley Armour, Danny Axford, Mark Basham, Thomas Connolley, Gwyndaf Evans, Katherine E. McAuley, Robin L. Owen, and James Sandy. A novel technique combining high-resolution synchrotron X-ray microtomography and X-ray diffraction for characterization of micro particulates. Meas. Sci. Technol., 22:115703, 2011.

[4] Eric Maire, Jérôme Adrien, and Philip J. Withers. In-situ X-ray tomographic study of materials. MDPI, 2020.

[5] Erika Tudisco, Clément Jailin, Arturo Mendoza Quispe, A. Tengattini, E. Andò, Stephen A. Hall, Gioacchino Viggiani, François Hild, and Stéphane Roux. An extension of digital volume correlation for multimodality image registration. Meas. Sci. Technol., 28(9), 2017.

[6] Narottam P. Bansal. Handbook of ceramic composites. Kluwer Academic Publishers, Boston, 2005.

[7] Vincent Mazars, Olivier Caty, Guillaume Couégnat, Amine Bouterf, Stéphane Roux, Sébastien Denneulin, Jérôme Pailhès, and Gérard L. Vignoles. Damage investigation and modeling of 3D woven ceramic matrix composites from X-ray tomography in-situ tensile tests. Acta Mater., 140:130-139, 2017.

[8] Myriam Berny, Thibaut Archer, Anne Mavel, Pierre Beauchêne, Stéphane Roux, and François Hild. On the Analysis of heat haze effects with spacetime DIC. Opt. Lasers Eng., 111:135-153, 2018.

[9] Léonard Turpin, Olivier Caty, Stéphane Roux, Sébastien Denneulin, and Eric Martin. Design of an in-situ multi-axial test to characterise the thermo-mechanical behavior of CMC parts. In JNC 21, pages 1-7, Bordeaux, France, 2019.

[10] José Baruchel, Jean-Yves Buffière, Eric Maire, Paul Merle, and Gilles Peix. X-Ray Tomography in Material Science. Hermes, 2000.

[11] A. Guvenilir, T. M. Breunig, J. H. Kinney, and S. R. Stock. Direct observation of crack opening as a function of applied load in the interior of a notched tensile sample of Al-Li 2090. Acta Mater., 45(5):1977-1987, 1997.

[12] Brian K. Bay, Tait S. Smith, D. P. Fyhrie, and M. Saad. Digital volume correlation: Threedimensional strain mapping using x-ray tomography. Exp. Mech., 39(3):217-226, 1999.

[13] Ante Buljac, Clément Jailin, Arturo Mendoza Quispe, Jan Neggers, Thibault Taillandier-Thomas, Amine Bouterf, Benjamin Smaniotto, François Hild, and Stéphane Roux. Digital volume correlation: Review of progress and challenges. Exp. Mech., 58(5):661-708, 2018.

[14] B. P. Wrobel. The evolution of digital photogrammetry from analytical photogrammetry. Photogramm. Rec., 13(77):765-776, 1991.

[15] Olivier Faugeras, Bernard Hotz, Hervé Mathieu, Thierry Viéville, Zhengyou Zhang, Pascal Fua, Eric Théron, Laurent Moll, Gérard Berry, Jean Vuillemin, Patrice Bertin, and Catherine Proy. Real time correlation-based stereo: algorithm, implementations and applications. Technical report, INRIA, Sophia Antipolis, France, 1993.

[16] Bin Chen, Haibo Liu, Bing Pan, and Bing Pan. Calibrating stereo-digital image correlation system using synthetic speckle-pattern calibration target. Meas. Sci. Technol., 31(9), 2020.

[17] Shaoyan Gai, Feipeng Da, and Ming Tang. A flexible multi-view calibration and 3D measurement method based on digital fringe projection. Meas. Sci. Technol., 30(2), 2019.

[18] A. King, N. Guignot, P. Zerbino, E. Boulard, K. Desjardins, M. Bordessoule, N. Leclerq, S. Le, G. Renaud, M. Cerato, M. Bornert, N. Lenoir, S. Delzon, J. P. Perrillat, Y. Legodec, and J. P. Itié. Tomography and imaging at the PSICHE beam line of the SOLEIL synchrotron. Review 
of Scientific Instruments, 87(9):093704-1-093704-8, 2016.

[19] Michael A. Sutton, Jean-José Orteu, and Hubert W. Schreier. Image Correlation for Shape, Motion and Deformation Measurements. Springer, 2009.

[20] Shaoyan Gai, Feipeng Da, and Xianqiang Dai. A novel dual-camera calibration method for 3D optical measurement. Opt. Laser Eng., 104(March 2017):126-134, 2018.

[21] Thibaut Archer, Pierre Beauchêne, Myriam Berny, and François Hild. Multi-instrumentation of very high temperature tests. In Residual Stress, Thermomechanics $\& 3$ Infrared Imaging, Hybrid Techniques and Inverse Problems, Volume 7, pages 73-76. Springer, 2019.

[22] Nobuyuki Otsu. A threshold selection method from gray-level histograms. IEEE Trans. Syst., Man, Cybern. Syst., SMC-9(1):62-66, 1979.

[23] Z Gao, Y Wang, G Gioia, and A M Cuitiño. A global approach for Digital Speckle Correlation. In Annu. Conf. Expo. Exp. Appl. Mech., number January, pages 1-4, 2002.

[24] Stéphane Roux, François Hild, Philippe Viot, and Dominique Bernard. Three-dimensional image correlation from X-ray computed tomography of solid foam. Compos. Part A, 39(8):1253-1265, 2008.

[25] Andrey N. Tikhonov and Y. Arsenin, Vasily. Solutions of Ill-Posed Problems. V. H. Winston \& Sons, 1977.

[26] Jinghao Yang, Zhenyuan Jia, Wei Liu, Chaonan Fan, Pengtao Xu, Fuji Wang, and Yang Liu. Precision calibration method for binocular vision measurement systems based on arbitrary translations and 3D-connection information. Meas. Sci. Technol., 27(10), 2016.

[27] Tomas Möller and Ben Trumbore. Fast, minimum storage ray/triangle intersection. J. Graph. Tools, 2(1):21-28, 1996. 